\title{
Glioblastoma de células gigantes: informe de un caso
}

\author{
Giant cell glioblastoma: case report.
}

\section{Beatriz Amalia Rivas, ${ }^{1}$ Luxely Mazlova Toledo, ${ }^{1}$ José Geovany García Herrera, ${ }^{2}$ Leonardo Flavio Medina Guillén, ${ }^{3}$ Allan Iván Izaguirre González. ${ }^{4}$}

${ }^{1}$ Médico Especialista en Anatomía Patológica; Departamento de Patología, Hospital Escuela,

${ }^{2}$ Médico Especialista en Neurocirugía; Hospital Nacional Mario Catarino Rivas, San Pedro Sula, Honduras,

${ }^{3}$ Médico en Servicio Social, Facultad de Ciencias Médicas, UNAH,

${ }^{4}$ Médico General; Maestrante de Epidemiología, Facultad de Ciencias Médicas, UNAH; Hospital General Santa Teresa, Región de Salud 3.

RESUMEN. Antecedentes: El Glioblastoma (GB) o astrocitoma grado IV, es un tumor agresivo que se origina de células gliales, con alto grado de malignidad, prevalencia menor al $1 \%$ en fosa posterior e incidencia menor al $0.5 \%$ de todos los GB. Actualmente se describen alrededor de 75 casos a nivel mundial. Descripción del caso clínico: Femenina, 24 años, referida a emergencia de Neurocirugía del Hospital Escuela Universitario, presentó cefalea holocraneana intensa, vómitos, náuseas, visión borrosa, vértigo y anorexia. Al examen neurológico mostró discreta adiadococinesia derecha y signos de papiledema. La tomografía axial computarizada cerebral evidenció lesión heterogénea en vermis extendido a hemisferio cerebeloso derecho, por lo que se realizó craniectomía suboccipital, abordaje transcerebelar, con citorreducción tumoral, encontrando masa vascularizada con componente quístico. Estudio anatomopatológico evidenció glioblastoma multiforme variante de células gigantes, confirmado con tinción de inmunohistoquímica (PFGA, CD34+ y vimentina). Paciente con buena evolución clínica postquirúrgica, egresada sin déficit neurológico. 16 meses después, presentó síndrome de recidiva tumoral y complicaciones, por lo que se reintervino en 4 ocasiones, posterior a recibir 30 dosis de radioterapia y 12 ciclos de quimioterapia, se reingresó con deterioro neurológico progresivo, signos meníngeos y síndrome de Parinaud, escala de Karnofsky (30 puntos), realizándose derivación ventrículo-peritoneal por compresión del IV ventrículo e hidrocefalia obstructiva secundaria, luego desarrolló neumonía intrahospitalaria, falleciendo a las dos semanas. Conclusiones: Es importante identificar la variante biológica del glioblastoma de forma temprana, para determinar pronóstico y acciones terapéuticas que influirán en la calidad de vida, así como la supervivencia.

Palabras clave: Cerebelo, Glioblastoma, Honduras, Neoplasias Primarias Secundarias, Proteína Ácida Fibrilar de la Glía.

Recibido: 03-05-2020 Aceptado para publicación 03-03-2021

Dirección para correspondencia: Dr. Allan Iván Izaguirre González

Correo electrónico: allanizaguirre9@gmail.com

Declaración de relaciones y actividades financieras y no financieras y conflictos de interés: ninguno.

\section{INTRODUCCIÓN}

El Glioblastoma (GB), llamado también astrocitoma grado IV, es un tumor agresivo que se origina de las células de la glía, poco diferenciado, con alto grado de malignidad, asociado invariablemente a un mal pronóstico. Solo el $33 \%$ de los pacientes sobrevive al año y el $5 \%$ viven más de 5 años tras el diagnóstico. ${ }^{1}$ Es el tumor primario del sistema nervioso central (TPSNC) más común y devastador, a pesar de que se ha incrementado la comprensión de sus fundamentos moleculares. ${ }^{2}$ La denominación multiforme se debe a la gran heterogeneidad que lo caracteriza con variados patrones y rasgos histológicos. ${ }^{3}$

La Organización Mundial de la Salud (OMS) clasifica los gliomas fundamentalmente por criterios histopatológicos en: astrocitomas, oligodendrogliomas, oligoastrocitomas y ependimomas. Tradicionalmente se han clasificado en dos subtipos morfológicamente idénticos: primarios (GB1) y secundarios (GB2). Además, establece una gradación basada en la célula del origen tumoral y características morfológicas relacionada con el pronóstico de la enfermedad, que identifica desde grado I (tumores circunscritos, de lento crecimiento y bajo potencial de conversión a un tumor de mayor malignidad) hasta grado IV (tumores de rápido crecimiento con alta tasa mitótica, neoformación de vasos sanguíneos y áreas de necrosis). ${ }^{1,4-6}$

En cuanto a su epidemiología, el GB en cualquiera de sus variantes se localiza más frecuentemente dentro de los hemisferios cerebrales, siendo, extremadamente rara su frecuencia cuando su localización está en fosa posterior (cerebelo), siendo su prevalencia menos del $1 \%$ (de todos los casos de GB en fosa posterior), y su variante celular gigante es muy infrecuente en adultos, representando tan solo el $0.15 \%$ de todos los tumores intracraneales. ${ }^{7,8} \mathrm{La}$ incidencia de los glioblastomas cerebelosos primarios es menos del $0.5 \%$ de todos casos $(20$ veces más bajos de lo esperado). ${ }^{4}$ El glioblastoma cerebelar $(\mathrm{GBc})$, es una entidad que sigue siendo poco conocida, debido a su incidencia relativamente baja. El primer caso de GB de fosa posterior se publicó en 1928, ${ }^{7}$ actualmente solo se describen alrededor de 75 casos en la literatura mundial. 


\section{DESCRIPCION DE CASO}

Femenina de 24 años de edad, raza mestiza, procedente de zona Nororiental de Honduras, referida a emergencia de Neurocirugía, del Hospital Escuela (H.E), en la ciudad de Tegucigalpa, Honduras, con historia de cefalea de 5 meses de evolución, en región occipital, intensa, opresiva, atenuada parcialmente con antiinflamatorios no esteroideos (AINES), de predominio matutino acompañada de vómitos precedidos de náuseas, 4 episodios y visión borrosa de 8 días de evolución, a su vez, vértigo subjetivo, labilidad emocional, anorexia. Paciente con antecedente personal patológico de fibroadenoma de mama izquierda BIRADS-II y síndrome de ovario poliquístico. Al examen neurológico se encontró discreta adiadococinesia derecha y presencia de papiledema grado II, sin alteración de la visión a colores y agudeza visual. El resto del examen físico y exámenes laboratoriales no mostraron alteraciones. Fue ingresada como síndrome de hipertensión endocraneana y síndrome cefalálgico, realizándose Imagen de Resonancia Magnética (IRM) cerebral, donde se aprecia lesión heterogénea en vermis cerebeloso, con extensión a hemisferio cerebeloso derecho (Figura 1A). Por lo que, se le realizó craniectomía suboccipital con remoción del arco posterior de $\mathrm{C} 1$, abordaje transcerebelar, con citorreducción tumoral, encontrando lesión blanco-grisácea, parcialmente aspirable, sin plano de clivaje, vascularizada, con componente quístico. Posteriormente se realizó TAC cerebral post quirúrgica inmediata donde se evidenció craniectomía suboccipital con sangrado residual (Figura 1B) e IRM cerebral que mostró recidiva tumoral (Figura $1 \mathrm{C}$ ). El estudio anatomopatológico evidenció proliferación neoplásica maligna de origen astrocitario, observándose áreas hipercelulares con fondo fibrilar y células tumorales pleomórficas de núcleos atípicos hipercrómicos y contándose hasta 5 mitosis anormales por campo de alto poder. Se observaron células tumorales gigantes multinucleadas (Figura 2A y $2 B$ ), diagnosticando hallazgos sugestivos de GB (grado IV según clasificación de OMS) en región fosa posterior-cerebelo. Se realizaron pruebas de marcadores de inmunohistoquímica con vimentina (1:50): Positivo (+++) citoplasmático (Figura 2C) y Proteína Fibrilar Glial Ácida [PFGA] (1:50): Positivo (+++) citoplasmático difuso en tumor (Figura 2D), CD34 (1:50): Positivo (+++) membranoso focal en células endoteliales, concluyendo Glioblastoma diagnosticado en abril de 2015. Paciente con buena evolución clínica post quirúrgica, fue egresada sin déficit neurológico. En enero de 2018, previo a 4 intervenciones neuroquirúrgicas por síndrome de recidiva tumoral y complicaciones (fístula LCR, infección del sitio quirúrgico), se realizó TAC cerebral que mostró edema perilesional (Figura 3A), componente quístico con compresión del IV ventrículo (Figura 3B) y dilatación del sistema ventricular con migración transependimaria (Figura $3 \mathrm{C}$ ), posterior a recibir 30 dosis de radioterapia y 12 ciclos de quimioterapia, se reingresó con deterioro neurológico progresivo, signos meníngeos y síndrome de Parinoud, escala de Karnofsky de 30 puntos. Se colocó derivación ventrículo peritoneal (DVP), sin embargo, desarrolló neumonía intrahospitalaria, falleciendo a las dos semanas.

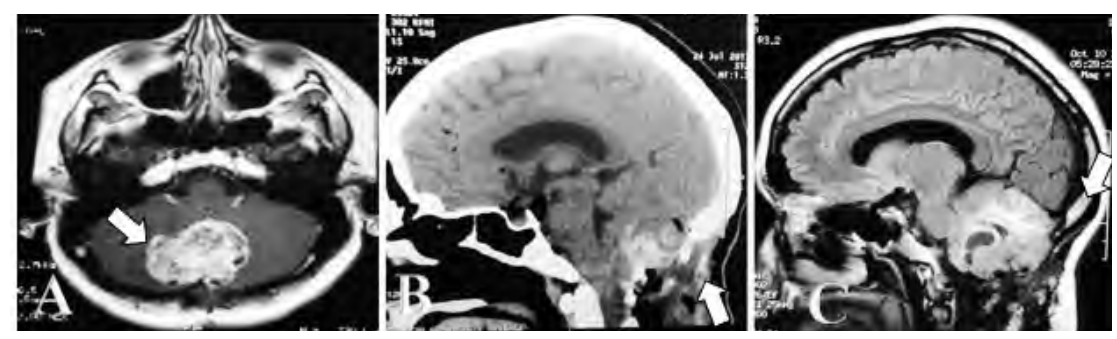

Figura 1. Estudios imagenológicos preoperatorios y postoperatorios. (A) Imagen de resonancia magnética (IRM) con gadolinio: se observa imagen hiperintensa en línea media, infratentorial, con extensión a ambos hemisferios cerebelosos, capta ávidamente medio de contraste. (B) TAC cerebral post quirúrgica inmediata, donde se evidencia craneotomía suboccipital, presencia de sangrado residual en lecho quirúrgico, así como en la región inferior de amígdalas cerebelosas. (C) IRM post quirúrgica donde se aprecia recidiva tumoral áreas heterogéneas con edema perilesional importante.
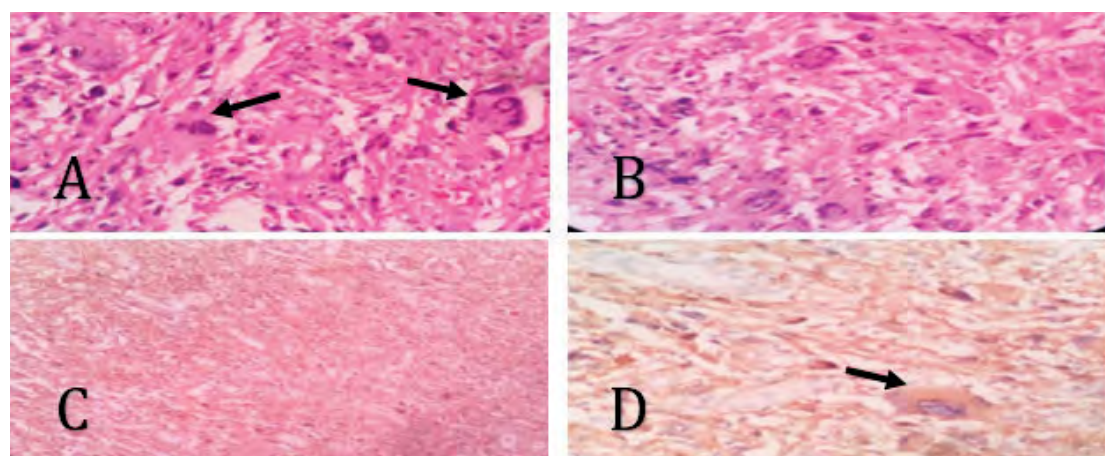

Figura 2. Imágenes anatomo-patológicas de cerebelo: (A) Tinción Hematoxilina-Eosina (10x) se observan células gigantes multinucleadas (flechas negras). (B) Múltiples células gigantes multinucleadas. (C) Tinción de Vimentina difusamente positivo (40x). (D) PFGA (Proteína fibrilar glial ácida) positiva con evidentes células gigantes, citoplasma y tejido glial (flecha negra).
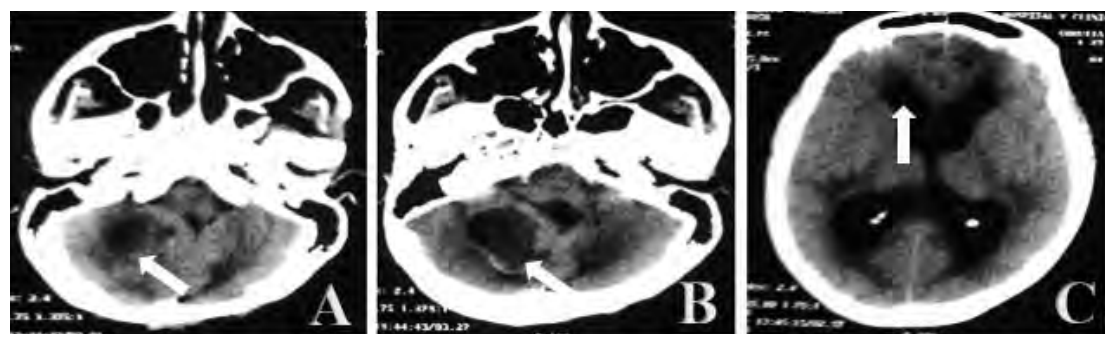

Figura 3. Tomografía Axial Computarizada, enero 2018 (Síndrome de recidiva tumoral): (A) Edema perilesional (flecha blanca). (B) Componente quístico con compresión del IV ventrículo (flecha blanca). (C) Dilatación del sistema ventricular con migración transependimaria (flecha blanca). 


\section{DISCUSIÓN}

El GB de células gigantes es un raro subtipo de GB que está presente en tan solo el $1 \%$ de los casos, siendo muy infrecuente y circunscrito, se caracteriza por el predominio de células multinucleadas gigantes enormes sobre una densa trama de reticulina. ${ }^{9,10}$ Normalmente se localizan con mayor frecuencia en los hemisferios cerebrales, pero al encontrarse en cerebelo, son extremadamente raros y van acompañados de un mal pronóstico, siendo su frecuencia hemisferios cerebelares $59 \%$, vermis $23 \%$ y solamente un $18 \%$ de forma mixta (vermis y hemisferios cerebelares). ${ }^{7,11}$

Afecta principalmente a los pacientes entre la quinta y sexta década de la vida (45-70 años), en dos tercios de los casos se presentan en adultos, teniendo una incidencia máxima a los 60 años $^{2}$ y es más común en hombres que en mujeres (proporción hombre/mujer 2:1), siendo menos frecuente en raza hispana. . $^{3,7,12}$ Estas características están presentes en este caso, ya que la variante de GB de células gigantes, se presentó en región de vermis con extensión a hemisferio cerebeloso derecho, volviendo la localización anatómica mucho más infrecuente, asimismo, la edad, género y raza de esta paciente, contrastan según la epidemiología y frecuencia de esta neoplasia.

El GB cerebeloso es de alguna manera diferente y a menudo dominado por los síntomas causados por el aumento de la presión intracraneal o la presión local sobre estructuras como el cuarto ventrículo, duramadre, o vasos sanguíneos: ataxia, desequilibrio, náuseas/vómitos y cefalea, ${ }^{2,7,13}$ además se pueden presentar convulsiones, hemiparesias y cambios en la personalidad. ${ }^{13}$ También tienen presentaciones atípicas como schwannomas vestibulares cuando alcanzan el ángulo pontocerebeloso (acompañado de tinnitus, desequilibrio progresivo, perdida neurosensorial y signo de Romberg positivo) y hemorragia cerebelosa hipertensiva, sin embargo, el patrón de presentación varía según el sitio del tumor y su velocidad de diseminación. 2,8,13,14 El cuadro clínico se caracterizó por cefalea en región occipital, intensa y progresiva, vómitos, anorexia, vértigo, labilidad emocional, visión borrosa, adiadococinesia derecha y papiledema grado II, mismos que concuerdan con los mencionados en la literatura respectivamente. La carcinogénesis representa un proceso de múltiples pasos complejos y se asocia con factores biológicos, químicos, físicos y genéticos. ${ }^{3}$ Existen muchos factores de riesgo estudiados y relacionados con la aparición de tumores cerebrales primarios, sin embargo, solo en algunos de ellos se ha podido establecer una relación real como la radiación ionizante, susceptibilidad genética, alergias (inversamente relacionadas al riesgo de aparición de gliomas) y metástasis tumorales secundarias (cáncer de pulmón, mama y piel), mismas que pueden metastatizar a región cerebelosa. ${ }^{15}$ Factores de riesgo que no se encontraron en esta paciente, a pesar de referir antecedente de fibroadenoma de mama izquierda BIRAD-II y síndrome de ovario poliquístico.

Entre los estudios de imagen a realizar se ha descrito la TAC con contraste puede ser útil para diferenciar la GB primaria de las metástasis. ${ }^{2}$ La resonancia magnética (RM) es el procedimiento diagnóstico de elección en el estudio de los tumores cerebrales, suele mostrar un centro necrótico con hipercaptación periférica que da aspecto de anillo (indicador clave para el diagnóstico de glioblastoma), además de detectar distintos niveles de vascularización en la parte externa del tumor. ${ }^{5}$ En cuanto a estudios histopatológicos, es rutina el análisis mediante biopsia para establecer el diagnóstico, además, los altos niveles de proteína fibrilar glial ácida (PFGA) en suero, se correlacionan con GB, mayor necrosis tumoral, edema perilesional y pruebas de inmunofluorescencia con $\mathrm{CD} 34+$ se ven asociadas a valores elevados de este mismo. ${ }^{16}$

La vimentina (una de las principales proteínas del citoesqueleto asociada a la estructura celular), al presentar valores elevados, proporciona un peor pronóstico y presencia de mayor capacidad tumoral invasiva (factores esenciales para la progresión del tumor). ${ }^{7,17,18}$ Estudios de imagen y anatomopatológicos (incluyendo marcadores de inmunohistoquímica) que se realizaron en esta paciente, reportaron concordancia con la localización anatómica, características morfológicas e histopatológicas (proliferación neoplásica maligna de origen astrocitario) compatible con GB de células gigantes en región cerebelosa.

Los diagnósticos diferenciales del GBC a considerar son metástasis de tumor primario extracraneal o secundario por neoplasia a distancia, absceso, infarto cerebelar, hemangioblastoma, linfoma primario de sistema nervioso central y astrocitoma anaplásico. ${ }^{2,3,7,8,19} \mathrm{~A}$ pesar de esto, las metástasis cerebelares se consideran usualmente en edades más avanzadas, además de ser necesario los antecedentes de cáncer en las regiones previamente descritas, ${ }^{15}$ los abscesos con neuro infecciones asociado a síndromes febriles y un infarto cerebelar normalmente tiene una clínica inespecífica, pero predomina la alteración en la marcha como síntoma más frecuente, seguido de vómitos, disartria y cefalea y la consideración especial de factores de riesgo vasculares (tabaquismo, hipertensión arterial, diabetes mellitus y dislipidemias), los hemangioblastomas implican normalmente una sintomatología cerebelosa (alteraciones de la marcha, mareo y ataxia) por edema tumoral y/o formación de quistes o síntomas debido a una hidrocefalia obstructiva, el cual, tanto por edad como por clínica sería descartable en comparación con un GB, además se debe tener como consideración que la hemorragia y necrosis tienen márgenes pobremente definidos presentes en el GB. ${ }^{20,21}$

La cirugía es la primera aproximación terapéutica (resección total), a pesar de que su característica infiltrante dificulte la resección completa del tumor. En general es imperativo la reducción tumoral lo más amplia posible, buscando reducir al mínimo el déficit neurológico, ya que se ha demostrado una correlación positiva entre la cirugía citorreductora y la supervivencia global. Posterior a la cirugía se continua con radioterapia y quimioterapia adyuvante. También se han considerado tratamientos inhibidores de la angiogénesis (bevacizumab y chlorotixin) el cual ha demostrado aumentar la supervivencia libre de progresión en pacientes GB de recién diagnóstico o recurrente, por la inhibición de la formación de vasos dependientes del factor 
de crecimiento endotelial vascular (VEGF) y la permeabilidad vascular en estos tumores altamente vasculares. ${ }^{2,10,12,22-25} \mathrm{El}$ manejo terapéutico quirúrgico se realizó por medio de una craniectomía suboccipital, abordaje transcerebelar, con cito reducción tumoral, que permitió encontrar una lesión blanco-grisácea parcialmente aspirable, vascularizada con componente quístico. A pesar de tener un deplorable pronóstico dentro de los tumores intracraneales (1-3 años), sobrevida de $33 \%$ al año y $3 \%$ a los 5 años, estas variables no están bien delimitadas para el GB en vermis cerebeloso, ya que la sobrevida promedio para ellos ha sido 8 meses, en donde solo el $21 \%$ llega al año y $2 \%$ a los 5 años, teniendo una menor sobrevida que otras localizaciones, siendo la esperanza de vida de aproximadamente 12-15 meses después del diagnóstico. ${ }^{2,12,14,15,18}$

En este pronóstico influyen factores como la resección parcial, edad mayor de 65 años, mayor tamaño de masa tumoral, invasión del tronco encefálico, falta de tratamiento adyuvante posterior a la cirugía citorreductora, son reconocidos como factores pronósticos de peor supervivencia. ${ }^{2}$ La raza asiática está asociada a mayor tiempo de sobrevida. ${ }^{25}$ Influyen también en el mal pronóstico las características infiltrativas del tumor y la resistencia de estos a la quimioterapia y radioterapia, lo que contribuye a la progresión de este. Algunas de estas características se presentaron en este caso, pese a las 30 dosis de radioterapia y 12 ciclos de quimioterapia, hubo presencia de invasión a tronco encefálico, teniendo un alto porcentaje de mortalidad a corto plazo (falleciendo a los 5 meses posterior al síndrome de recidiva tumoral).

Las complicaciones son debido al tratamiento tanto citorreductor por el riesgo de infección postquirúrgica como citotóxico (radioterapia y quimioterapia) que predisponen a neutropenia, neumonitis y sobreinfecciones. ${ }^{26-28}$ Dentro de las reintervenciones quirúrgicas mencionadas anteriormente, se presentaron complicaciones asociadas que incluyeron fístula de LCR e infección del sitio quirúrgico respectivamente. Pese a que se realizó el abordaje terapéutico adecuado, estas mismas pudieron influir y relacionarse a la causa básica de muerte, neumonía intrahospitalaria.

\section{CONTRIBUCIONES}

$B R$, LT, proporcionaron las imágenes histopatológicas, inmunohistoquímica y biopsia. JG, proporciono los estudios imagenológicos (TAC, IRM). LM, Al, obtuvieron las imágenes histopatológicas, inmunohistoquímica y radiológicas. Todos los autores participaron de la concepción de la idea del caso clínico, búsqueda y elaboración de referencias bibliográficas, discusión, redacción y revisión del manuscrito final.

\section{AGRADECIMIENTOS}

Agradecemos al personal del área de archivo del Hospital Escuela por la búsqueda y acceso al expediente clínico.

\section{REFERENCIAS}

1. Sierra Benítez EM, León Pérez MQ, Laud Rodríguez L, Carrillo Comas AL, Pérez Ortiz L, Rodríguez Ramos E. Gliomas malignos: biología molecular y detalles oncogenéticos. Rev Méd Electrón [Internet]. 2018 [consultado 24 junio 2018]; 40(4). Disponible en: http://www.revmedicaelectronica.sld. cu/index.php/rme/article/view/2480/3973

2. Gao S, Liu X, Cheng P, Yuan X, Niu J, Bai Y, et al. A Primary Cerebellar Glioblastoma Multiforme Mimicking Vestibular Schwannoma. J Craniofac Surg. 2016;27(7): e623-e626.

3. Castañeda CA, Casavilca S, Orrego E, García-Corrochano P, Deza P, Heinike $\mathrm{H}$, et al. Glioblastoma: análisis molecular y sus implicancias clínicas. Rev Perú Med Exp Salud Publica. 2015;32(2):316-25.

4. Agarval A, Bhake A, Kakani A, Hiwale KH, Khairul Enam Sk. Cerebellar glioblastoma multiforme in an adult. J Cancer Res Ther. 2014;10(3):777778.

5. Gómez-Útero Fuentes E, Navarro Expósito F, López González JL, Lamarca LA, Álvarez-Mon Soto M. Actualización en tumores del sistema nervioso central. Medicine. 2013;11(24):1468-83.

6. Louis DN, Perry A, Reifenberger G, Deimling AV, Figarella-Branger D, Cavenee WK, et al. The 2016 World Health Organization Classification of Tumors of the Central Nervous System: a summary. Acta Neuropathol. 2016; 131:803-820

7. Sciacero P, Franco Girelli G, Cante D, Franco P, Casanova Borca V, Grosso $\mathrm{P}$, et al. Cerebellar glioblastoma multiforme in an adult woman. Tumori. 2014; 100(3): e74-e78.

8. Kikuchi K, Hiratsuka Y, Kohno S, Ohue S, Miki H, Mochizuki T. Radiological features of cerebellar glioblastoma. J Neuroradiol. 2016;43(4):260-265.

9. Oh T, Rutkowski MJ, Safaee M, Sun MZ, Sayegh ET, Bloch O, et al. Survival outcomes of giant cell glioblastoma: Institutional experience in the management of 20 patients. J Clin Neurosci. 2014:21(12);2129-34.

10. Ortega-Aznar A, Jiménez-León $P$, Martínez E, Romero-Vidal FJ. Aspectos clínico-patológicos y moleculares de valor diagnóstico y pronóstico en gliomas. Rev Neurol. 2013; 56:161-70.

11. Picart T, Barritault M, Berthillier J, Meyronet D, Vasiljevic A, Frappaz D, et al. Characteristics of cerebellar glioblastomas in adults. J Neurooncol. 2018;136(3): 555-563.

12. Alegría-Loyola MA, Galnares-Olalde JA, Mercado M. Tumores del sistema nervioso central. Rev Med Inst Mex Seguro Soc [Internet]. 2017[consultado 14 abril 2020]; 55(3):330-40. Disponible en: https://pubmed.ncbi.nlm. nih.gov/28440987/

13. Linsenmann $\mathrm{T}$, Monoranu $\mathrm{CM}$, Westermaier $\mathrm{T}$, Varallyay $\mathrm{C}$, Ralf-Ingo $\mathrm{E}$, Vince GH. Exophytic Glioblastoma Arising from the Cerebellum: case report and Critical Review of the Literature. J Neurol Surg A Cent Eur Neurosurg. 2013;74(4):262-264.

14. Behaine JM, Aristizábal JH. Hipertensión endocraneana. En: Vélez van Meerbeke A, Uribe Granja MG, Prada Gaviria DM. Decisiones en neurología Tomo II. Bogotá: Editorial Universidad del Rosario; 2016.p.35-53.

15. Contreras LE. Epidemiología de tumores cerebrales. Rev Méd Clín Condes. 2017;28(3):332-338.

16. Mei X, Chen YS, Chen FR, Xi SY, Chen ZP. Glioblastoma stem cell differentiation into endothelial cells evidenced through live-cell imaging. Neuro Oncol. 2017; 19(8):1109-1118.

17. Tichy J, Spechtmeyer S, Mittelbronn M, Hattingen E, Rieger J, Senft C, et al. Prospective evaluation of serum glial fibrillary acidic protein (GFAP) as a diagnostic marker for glioblastoma. J Neurooncol. 2016;126(2):361-9.

18. Zhao J, Zhang L, Dong X, Liu L, Huo L, Chen H. High Expression of Vimentin is Associated with Progression and a Poor Outcome in Glioblastoma. Appl Immunohistochem Mol Morphol. 2018;26(5):337-344.

19. Araneda P, Sujima E, Paredes-Manzo P, Vallejo R, Valdivia F, Sinning M, et al. Glioma difuso de línea media H3K27M positive en adulto. Caso clínico. Rev Med Chile. 2019; 147:1487-1490.

20. Silva D, Grabowski MM, Juthani R, Sharma M, Angelov L, Vogelbaum MA, et al. Gamma Knife radiosurgery for intracranial hemangioblastoma. J Clin Neurosci. 2016; 31:47-51.

21. Khan AA, Murphy M, Tumours of the central nervous system. Surgery.2015;33(8):369-376.

22. Linsenmann T, Monoranu CM, Vince GH, Westermaier T, Hagemann C, 
Kessler A, et al. Long-term tumor control of spinal dissemination of cerebellar glioblastoma multiforme by combined adjuvant bevacizumab antibody therapy: a case report. BMC Res Notes. 2014; 7:496.

23. Rivero-Morey RJ, Ramírez-Morfa CA, Rivero-Morey J. Tratamiento del glioma cerebral de alto grado en el paciente adulto. Univ Méd Pinareña [Internet]. 2020 [citado: 25 de julio 2018]; 16(1): e389. Disponible en: http:// www.revgaleno.sld.cu/index.php/ump/article/view/389

24. Cohen-Inbar O, Zaaroor M. Glioblastoma multiforme targeted therapy: The Chlorotoxin story. J Clin Neurosci. 2016; 33:52-58.

25. Adams H, Chaichana KL, Avendaño J, Liu B, Raza SM, Quiñones-Hinojosa A. Adult Cerebellar Glioblastoma: Understanding Survival and Prognostic Factors Using A Population-Based Database from 1973 - 2009. World Neuros. 2013;80(6): e237-e243.

26. Garrell Lluís I, Gimferrer Artigas N, Marzo-Castillejo M. Los efectos adversos de la radioterapia oncológica. ¿Qué debe saber el médico de familia? FMC. 2015; 22(10):554-63.

27. Garrell Lluís I, Gimferrer Artigas N, Marzo-Castillejo M. Efectos adversos de la quimioterapia, las terapias dirigidas contra el cáncer y la hormonoterapia: ¿qué debe saber el médico de familia? FMC. 2015;22(9):482-93.

28. Das S, Philip A. Angiogénesis in Glioblastoma. N Engl J Med. 2013;369(16): 1561.
ABSTRACT. Background: Glioblastoma (GB) or grade IV astrocytoma, is an aggressive tumor that originates from glial cells, with a high-grade of malignancy, prevalence of less than $1 \%$ in the posterior fossa and incidence of less than $0.5 \%$ of all GB. There are currently about 75 cases described worldwide. Clinical case description: 24-year-old female, referred to HEU's Neurosurgical emergency, presented intense holocrine headache, vomiting, nausea, blurred vision, vertigo, and anorexia. The neurological examination showed discrete right adiadokinesia and signs of papilledema. Brain CT showed heterogeneous lesion in vermis extended to right cerebellar hemisphere, therefore, suboccipital craniectomy with a transcerebellar approach, was perfomed, leading to tumor cytoreduction, finding vascularized mass with cystic component. Anatomopathological study showed glioblastoma multiforme variant of giant cells, confirmed with immunohistochemical staining (GFAP, CD34+ and vimentin). Patient with good post-surgical clinical evolution, is discharged without neurological deficit. At 16 months later she presents tumor recurrence syndrome and complications, was re-intervened on four occasions, after receiving 30 doses of radiotherapy and 12 cycles of chemotherapy. She was readmitted with progressive neurological deterioration, meningeal signs and 30-point of Karnofsky scale and Parinaud's syndrome and underwent PVS by IV ventricular compression and secondary obstructive hydrocephalus, performing ventricle-peritoneal shunt by compression of the IV ventricle and secondary obstructive hydrocephalus, then he developed intrahospital pneumonia, dying after two weeks. Conclusions: It's important to identify the biological variant of glioblastoma early, to determine prognosis, therapeutic actions that will influence quality of life, as well as survival.

Keywords: Cerebellum, Glioblastoma, Glial Fibrillary Acidic Protein, Honduras, Neoplasms, Second Primary. 